\title{
2. X-RAY BINARIES AND COMPACT OBJECTS
}

P.J.N. DAVISON / Periods in X-Ray Sources

A.P. WILLMORE / Recent Transient X-Ray Sources

R. NOVICK, H.L. KESTENBAUM, K.S. LONG, E.H. SILVER, M.C. WEISSKOPF, and R.S. WOLFF / OSO-8 X-Ray Polarimeter and Bragg Crystal Spectrometer Observations

J.F. DOLAN, B.R. DENNIS, C.J. CRANNELL, K.J. FROST, and L.E. ORWIG / OSO-8 Observations of Cygnus XR-1

G.W. CLARK / X-Ray Bursts

J.E. GRINDLAY / Globular Cluster X-Ray Sources

R.N. HENRIKSEN / A Model for Bursting X-Ray Sources

F.K. LAMB, A.C. FABIAN, J.E. PRINGLE, and D.Q. LAMB / Bursting $X$-Ray Sources: A Theoretical Framework for Accretion Models

L. MARASCHI and A. CAVALIERE / X-Ray Bursts of Nuclear Origin?

J.B. HUTCHINGS / Optical Observations of X-Ray Binaries

Y. AVNI / Masses of Compact X-Ray Sources

E.P.J. VAN DEN HEUVEL and G.J. SAVONIJE / The Evolutionary History of X-Ray Binaries

R. MCCRAY / ACcretion Flows in Binary $\mathrm{X}$-Ray systems

Y. KONDO / Coordinated Campaign to Observe X-Ray Binaries 


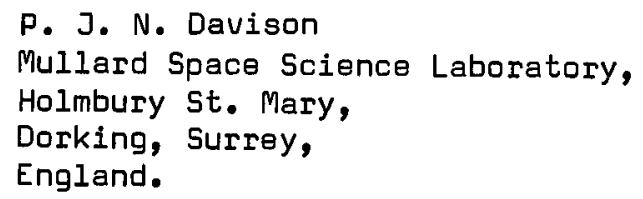

\section{INTRODUCTION}

Known periodicities in $X$-ray sources cover a wide range of values a factor of $10^{8}$ or $10^{9}$ from the $33 \mathrm{mS}$ of the Crab pulsar up to 35 days for the Her $X-1$ repeat of its up state, or even years if one believes in the not inconceivable idea that at least one "transient" (perhaps not yet even discoveredd) is exhibiting some form of repetitive behaviour. These periodicities are not just astronomical curiosities to keep theoreticians busy speculating. They do have several solid uses which the mere experimenter can take advantage of. These uses do, inevitably overlap, and vary in importance according to whom one is speaking. In the short space available I will concentrate on recent measurements on $X$-ray sources showing Doppler modulated periodicities, and on sources which may well show such changes when they are studied more closely.

\section{PROPERTIES OF SPECIF IC SOURCES}

\section{$3 \cup 0115-73$}

SMC $X-1$ was first reported to be an eclipsing binary system by Schreier et al. (1972). Tuohy and Rapley (1975) derived an improved period of 3 de9217 \pm 0 d00012, in excellent agreement with the optical period of $3.8921 \pm 0.0005$ reported by Petro et al. (1973) for the star Sanduleak 160, which had been suggested by webster et al. (1972) as the optical counterpart.

Probably the most important measurement on SMC $X-1$ since the early observations was the detection of coherent pulses with a period of 0.716 s (Lucke et al 1976). This immediately raised the possibility of making a Doppler shift determination of the mass function of the system, which combined with optical data allows one to make estimates of interesting parameters of the system.

Such a measurement has now been made by the SAS-3 instrument 
(Primini et al. 1976); it was found that vsini $=301.5 \pm 4.0 \mathrm{~km} \mathrm{~s}-1$, and $\theta<0.04$. Combining the SAS-3 zero phase with that given by Tuohy and Rapley (1975) leads to an average orbital period of 3 d89264 \pm 0 doo0 28 over the period August 1974 to February 1976.

The mass function given by the SAS-C observations is $11.05 \pm 0.22 M_{\odot}$. As noted by Primini et al, without information on orbital inclination $i$ and the mass ratio $q\left(=M_{x} / M_{0}\right)$, all that can definitely be said is that Sk160 has a mass greater than $\sim 11 M_{\odot}$. However, the optical Doppler curve, and the $X$-ray eclipse duration do allow one to place limits on the ranges of $i$ and $q$, (Wilson and Wilson 1976; Avni and Bahcall 1976). The mass ratio is determined from the ratio $K_{0} / K_{X}$, where $K$ is the projected orbital velocity in question. Due to the somewhat uncertain knowledge of $K_{0}$, $q$ is not well determined, and Primini et al. give $0.07<q<0.2$.

Wilson (1972) has derived the mass ratio for the Cen $X-3$ system from a knowledge of the eclipse duration; following the same lines, and adopting an eclipse duration of 0.60 (a semi duration of 2787), Primini et al. have placed the mass of the $X$-ray object in the range 1.1 to $4.0 \mathrm{M}_{\odot}$, and the mass of $5 k 160$ in the range 15 to $22 \mathrm{M}_{\odot}$.

What do we need to investigate in the future for this system? Well, $\theta \theta$ is a critical parameter as far as the $X$-ray work is concerned. A relatively small change in $\theta$, by 2 - $3^{\circ}$ changes $q$ by 50 to $100 \%$ depending on the inclination chosen. Apart from theoretical questions common to all the binary sources, probably the most important and useful thing to do would be to improve our knowledge of the optical projected velocity, which is rather poorly defined at present, so as to further restrict the allowed ranges for the system masses. As far as further $X$-ray measurements are concerned, more Doppler measurements of the pulsation frequency will be useful for defining any period changes, and to establish more precise values of the orbital period by measuring phase zero accurately. The projected velocity too can be monitored for any secular or periodic changes.

\section{$300352+30$}

Giacconi et al. (1974), first suggested that this source, with a $2^{\prime} \times 14^{\prime}$ error box, may be associated with the 6th mag. Be star $X$ Persei. This association has been greatly strengthened by Copernicus measurements. (Hawkins et al. 1975; Mason et al. 1976b).

A temporal analysis of the Copernicus $X$-ray data showed a strong peak in the power spectrum at a period of $13.9 \mathrm{~min}$ (White et al. 1976c). The secular behaviour of the period is shown in figure 1. There is a small but possibly significant difference between the 1974 Feb and 1975 Jan and 1976 Jan periods, which are derived from longer spans of data than the earlier measurements. In December 1972 measurements were also made in the 0.6 to $1.9 \mathrm{keV}$ and 1.0 to $3.1 \mathrm{keV}$ energy bands, and a 13.9 minute periodic component was detected. The Copernicus data also 


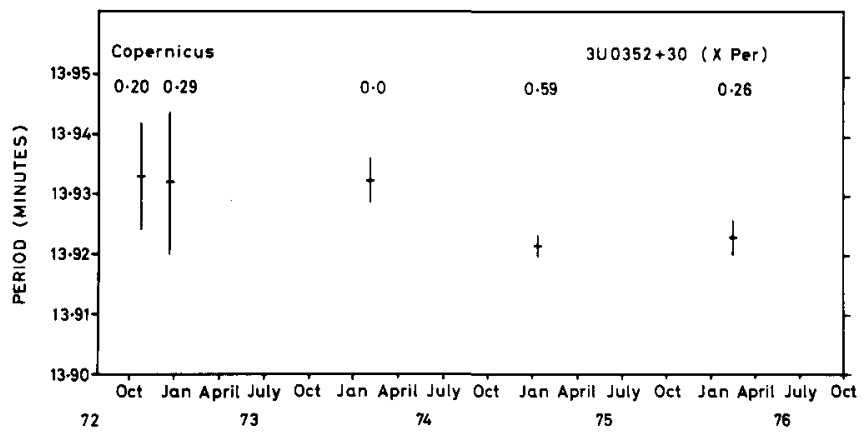

Figure 1. $X$-Perseus, period versus time.

showed that there might be a longer period modulation, at either 11.2 hours or 22.4 hours period. An Ariel 5 observation in August 1975 did show count rate maxima separated by about 22 hours, although the source was very variable; long continuous observations will be needed to overcome the effects of the intrinsic variability of the source.

Turning now to the optical counterpart, and the topic of most concern to this meeting, namely periods. Margon et al. (1976) carried out optical photometry simultaneously with the OAO X-ray measurements, and failed to detect any covariability. Any 22 hour period would have been seen if it had amplitude 0.01 mag. or greater (cf HD226868, .05 mag; Cen X-3, $0.12 \mathrm{mag}$ ). Robinson and Africano (1975) have analysed an $8 \mathrm{hr}$. stretch of data taken through a $U$ filter, and concluded that any $13.924 \mathrm{~min}$. periodicity had an amplitude of less than $4 \times 10^{-4}$ magnitude; on the other hand, Liller (1975b) has folded two 1 hour stretches of data, separated by 1 day, at a period of 13.924 minutes and has found a double sine curve with full amplitude of $0.89 \pm .10 \%$.

Hutchings et al. (1974) have investigated the velocities of emission and absorption features in $X-P e r$, and have made searches for periodicities in the range 2 - 800 days. All groups of data showed best periods in the range 575 - 595 days. The mass function they derived was $18.2 \pm 3.5 \mathrm{M}_{\odot}$; they also argued that the system may be a widely separated binary with a primary of mass $\sim 20 M_{\odot}$ and a secondary of mass $\sim 40 M_{\odot}$ which is a weak $X$-ray emitter. The measured velocities were $\sim 60 \mathrm{~km} / \mathrm{sec}$ and $\sim 10 \mathrm{~km} / \mathrm{sec}$; it is instructive to ask if these velocities would be detectable via a Doppler shift in the frequency of the 13.9 min. period. The half amplitude of period variation is $3 \times 10^{-3}$ min. for vsini $=60 \mathrm{~km} / \mathrm{sec}$. This is slightly larger than the accuracy quoted for the Jan 1975 period measurement $\left(2 \times 10^{-3}\right.$ min.). Thus a small improvement in the accuracy of the period measurement, coupled with a sufficient number of samples over the suggested 584 day orbital period should lead to further understanding of this system. On the other hand, if the $X$-ray source is associated with the more massive star 
(as suggested by Hutchings et al. 1974), the low orbital velocity of $10 \mathrm{~km} / \mathrm{sec}$ will lead to a period half amplitude of only $5 \times 10^{-4}$ minute a number which is substantially below the practical limits of present instrumentation. Figure 1 indicates the 584 day phase for the Copernicus measurements.

\section{$A 0535+26$}

This transient-type source was first detected by the rotation modulation collimator experiment on the satellite Ariel 5 (Rosenberg et al. 1975), during routine observations of the Crab Nebula. A periodic signal was observed at $104.14 \pm 0.16 \mathrm{sec}$ by Ariel 5 on 1975 April 28th. SAS-3 subsequently made a determination of the period as 103.8274 \pm .0004 seconds averaged from May 30 to June 2, 1975 (Bradt et al. 1976) Bradt et al. also determined that a constant period was an acceptable fit to the 3 day span of data. The source was observed to brighten again after its original outburst and decay, and SAS-3 was again able to detect pulsations and measure their period (Rappaport et al. 1976a); a remanalysis was made of the May/June data, using only the lower energy channels $(1.2-6 \mathrm{keV})$. Pulse arrival times, relative to the best fit constant pulse period for the two sets of data are shown in Fig.2 (Copyright: the Astrophysical Journal, reproduced with permission).

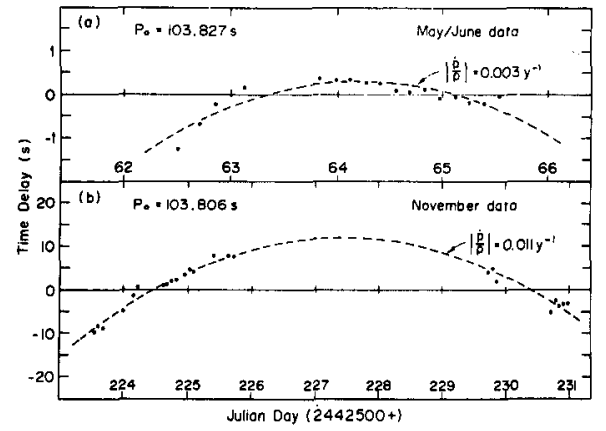

Figure 2. A0535+26, pulse arrival times.

The two sets of data were combined to search for allowed circular orbits (which is one way of explaining the data), assuming a constant intrinsic pulse period. Several acceptable ranges of orbital periods occured. A lower quality SAS-3 observation on 1975 July 26th reduces the likelihood of several of the possible period being correct, while the tentative association of the $X$-ray source with the OB star HDE 245770 (Liller 1975a) indicates that orbital periods less than 35 days (mass function $<1 M_{\odot}$ ) are also unlikely. It was therefore concluded that the most likely orbital periods are $39^{d} \pm 0 d 5$ and $77^{d} \pm 1 d$, with mass functions $1 M_{0}$ and $8 \mathrm{~m}_{\odot}$ respectively. These conclusions depend critically on the assumption that there was no change in the intrinsic pulse period either during the SA5-3 observations or between them. The period measured by Ariel 5 on 1975 April 28 was not consistent with any of the orbits deduced from the SAS-3 data, but Rappaport et al. suggest that this may be due to the occurrence of a spinup when the source was near maximum 
luminosity. Rappaport et al. believe that the most plausible model for this system is a compact object in a wide orbit around an $\mathrm{OB}$ star with a variable stellar wind - accretion of the wind onto the compact object producing the $X$-rays. Obviously, because of the various assumptions made in arriving at the above conclusions, this source must be thoroughly investigated if it becomes strong enough once more. In the meantime, however, there is great scope for detailed study of the suggested optical candidate. Schmidt and Romanishin (1975) have reported a $3 \sigma$ upper limit of $0.2 \%$ for any variation in intensity at a period of 104 seconds, during observations made near the November $197 \overline{5}$ X-ray maximum. However, no reports have yet been made of any search for the velocities of any absorption or emission features in the stellar spectrum; such a search may yield as fruitful a result as has the similar study of $X$-Per.

$300900-40$

3u0900-40 was discovered in rocket observations by Chodil et al. (1967), and was subsequently found in 050-7 satellite observations to be an eclipsing binary with an orbital period of about 9 days (Ulmer et al, 1972). Positional data from Uhuru led to identification of an optical counterpart, HD77581, a BD supergiant. A mass function was then derived from the optical star. SAS-3 observations in 1975 showed that the $X$-ray star exhibited regular pulsations with a 283 second period (McClintock ot $a l, 1976$ ) and that this was clearly modulated by the Doppler shift as the compact object moved in its 9 day orbit. The orbital parameters which the SAS-3 group derived (Rappaport et al. 1976b) include vsini $=273 \pm 9 \mathrm{~km} \mathrm{~s}-1$, mass function $=18.5 \pm 1.6 \mathrm{~m}_{\text {}}$ and $\theta=0.126 \pm 0.041$.

An interesting feature of this system is that it has a small but significant orbital eccentricity, unlike the other systems for which orbital parameters are measureable (SMC $x-1$, Cen $x-3$, Her $x-1$ ).

The most recent optical study of the companion star has given a projected orbital amplitude of aoptsini $=8.0 \pm 0.7$ It sec (van Paradijs et al. 1976). Rappaport et al. have refitted the optical data using the values of $e$ and $\omega$ derived from the $x$-ray data and have got the result a optsini $=7.79 \pm 0.74$ It sec. Other reported measurements yield values of a optsini in the range 8 - 16 light sec. Adopting the value of 7.79 It sec will give the smallest stellar masses, and the SAS-3 results show that $M_{x} \sin ^{3}=1.45 \pm 0.16 M_{0}$ and $M_{0 p t} \sin ^{3} i=21.1$ $\pm 0.9 M_{\odot}$. The inclination is probably no less than $\sim 70^{\circ}$, as indicated by an analysis of the ellipsoidal light variations of the star, and the duration of the $X$-ray eclipses (Auni and Bahcall 1975), so the lower limit to the $X$-ray object's mass is close to $1.4 \mathrm{M}_{\odot}$. This is a critical mass in theoretical considerations, so it is of great importance to make more accurate measurements of the optical radial velocities and their variations. 
It may well be possible to follow the motion of the line of apses in this system, and we can easily make a crude estimate of how long an impatient $X$-ray astronomer will have to wait for results. Using the formula given by Sterne (1939) for the ratio of orbital period to apsidal motion periods, we arrive at the approximate relation for the 3L0900-40/HD 77581 system

$$
\text { Paps } \simeq \frac{5.5 \text { Porh }}{\text { kopt }}
$$

kopt can be calculated according to various models, but looking at the range of values observed in practice (wood, 1963), we see that $k$ may well lie in the range $2 \times 10^{-3}$ to $2 \times 10^{-2}$. In other words, we can expect Paps to lie in the range 60 yrs to 6 yrs. If we take the calculations of stothers (1974) as a guide for a star of mass $20 \mathrm{M}_{0}$, we get Paps $\sim 17$ years, with a consequent movement of the line of apses through the full $\pm 95 \%$ error band in little more than 2 years.

$3 \sqcup 1223-62$

This object, also referred to as GX301-2, was found to exhibit regular pulsations by white et al. (1976a), with a period of about 11.6 minutes. Period measurements during 1975 and early 1976 are available from 4 different $X$-ray satellites, and the values found are shown in Figure 3. There is no doubt that the pulse period has undergone changes; what we see in the figure are undoubtedly intrinsic changes, rather than Doppler derived, since the latter hypothesis would lead to an extremely unlikely mass function.

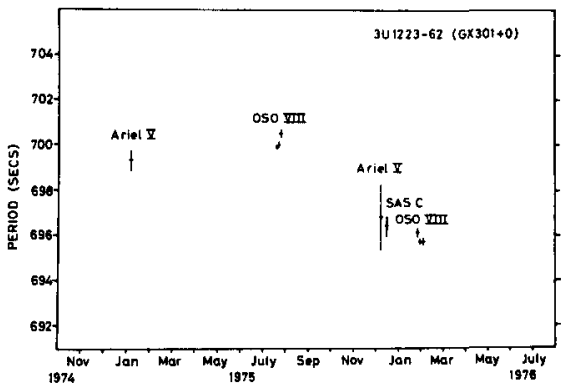

Figure 3. 3U1223-62, period versus time.

The star URA 977 was suggested by Vidal (1973) and Jones et al. (1974) as a possible counterpart of the $X$-ray source, and the recent observation of optical pulsations by Mauder (1976) shows that the suggestion was correct. Mauder (1974) has presented evidence for a photometric variation of period 13.5 , while Bord et al. have found evidence for other periods in the range 5 to 20 days. The latter authors concluded however that the periodic variations were not statistically significant. Nevertheless it will be interesting to see if the 0SO-8 
data, which spans 8 days (Swank et al. 1976), shows any evidence for orbital motion of the X-ray source. Whatever the outcome of such an investigation, it is clear that, as for several other periodic sources, an investigation of the time behaviour of the radial velocity of optical spectral lines is the best way of identifying any orbital period, in the face of the large changes in the intrinsic $X$-ray pulsation period.

\section{$3 \cup 1653+35$}

Hercules $X-1$ and its optical companion $H Z$ Her were one of the first $X$-ray binary systems to be recognised; both objects have been extensively studied. Nevertheless there are still a number of incompletely understood phenomena relating to the system which are worthy of further experimental and theoretical study.

Although the early Uhuru results indicated that the $X$-ray source was visible from Earth for an approximate 12 day continuous span (apart from the 1.7 day eclipses), and was invisible for the remainder of the 35 day cycle, an observation by Fabian et al. (1973) using Copernicus data showed a clear active state approximately half way between successive turn ons in the 35 day cycle. Chevalier and Ilovaisky (1974) reported a period of optical activity in the $U$ band, which was coincident in time with the Copernicus observation. Further observations of this so called anomalous on state were made by the Sky Survey experiment on Ariel 5 (Cooke and Page 1975), and the Uhuru group (Jones et al. 1976) have now been able to find in their data observations showing the presence of X-ray emission throughout the 35 day cycle, with enhanced emission midway through the cycle. Ariel 5 observations (Davison and Fabian 1976) also showed the presence of emission at a level of $4 \%$ of the peak emission for at least 2 days prior to turn on. Early Uhuru data showed that the time of occurrence of a pre-eclipse dip marched backwards.relative to the 1.7 day orbital eclipse. This phenomena also occurred in the Copernicus data. Fabian and Davison (1974) noted that the repeat time between pre-eclipse dips was 1.62 days, and that this period would beat with the 1.7 day period to give a value of 34.88 days. The 1.62 day period is also present in the optical power density spectrum of Deeter et al. (1976).

An interesting feature which is emerging from the combined effort of many groups is the long term behaviour of the 35 day cycle. Figure 4 shows reported turn on times plotted relative to an assumed constant repeat time of 34.95 days. There is a hint of quasi-regular behaviour here, but it is possible that such an effect could be due to a random walk in D-C. We must wait and see if this pattern continues.

The Uhuru observations showed a gradual decrease in the 1.24 second pulsation period, with significant departures from a steady monotonic decrease. The only reported measurement with useful accuracy that has been made since the Uhuru work comes from Ariel 5 (Davison and Fabian 1976). The value derived, $1.237796 \pm 0.000008$ seconds continues the average downward trend. This period is that measured at JD 2442482.292 


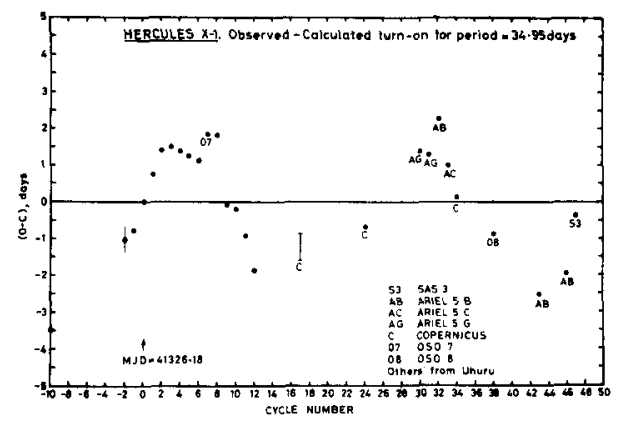

Figure 4. Her $X-1$ turn-on times of 35 day cycle.

\pm 0.004 , which is our epoch of zero phase (centre of the 1.7 day eclipse). The zero phase differs from the extrapolated Uhuru value by $7+6$ minutes, assuming a constant orbital period of 1.700165 .

Although our zero phase epoch agrees with the Uhuru value, we do find a small difference in the measured values of $v_{x} s i n i$, our value of $163.8 \pm 2.1 \mathrm{~km} \mathrm{~s}^{-1}$ being slightly lower than the $169.0 \pm 0.1 \mathrm{~km} \mathrm{~s} \mathrm{~s}^{-1}$ given by the Uhuru data. The errors here are equivalent to a 68 percent confidence level. If this difference is not just a statistical fluke it could be explained by a change in the apparent orbital inclination, due to a precession of the orbit plane caused by differences in direction between the orbital angular momentum axis and the rotation axis of $\mathrm{HZ}$ Her.

Much optical work has now been done on the star $\mathrm{HZ} \mathrm{Her.} \mathrm{One} \mathrm{of}$ the interesting features to study would be the radial velocity versus orbital phase so as to combine with the $X$-ray values and get the masses out. There do appear to be difficulties here though, caused in part by the effect of the rotation of the (non uniformly bright) star on the velocities and shape of the emission lines. However, Middleditch and Nelson (1976) have made an extensive study of the 1.24s optical pulsations, and have developed a model which gives a direct determination of the mass ratio $M_{H Z}$ Her $/ M_{H e r} x-1$ as $1.68 \pm 0.10$. In addition, and most importantly, they show that $\mathrm{HZ}$ Her fills its Roche Lobe, and so use of the measured $X$-ray eclipse duration in determining the masses is probably valid. The values which they calculate are $i=87^{\circ} \pm 3^{\circ}$, $M_{H e r} X-1=1.30 \pm 0.14 \mathrm{M}_{\bullet}$ and $M_{H Z}$ Her $=2.18 \pm 0.11 \mathrm{M}_{\bullet}$.

$3 \cup 1728-24$

The X-ray source GX1+4 was first identified by Lewin et al. (1971) from data obtained during a balloon flight. The data showed a possible variability with a period of $\sim 138$ sec. White et al. (1976a) announced that Copernicus observations showed that the low energy $X$-ray source was pulsing with a period of 4.3 minutes, though it was recognised (White et al. 1976b) that this may be an alias of a modulation below 
the instrument Nyquist frequency (2.9 minutes). The most likely true period would then be close to 130 seconds. Observations from SAS-3 (Lewin et al. 1976b) and 0SO-8 (Becker et al. 1976) have amply confirmed the presence of a periodic modulation of the flux, and have also confirmed the decrease in period which was suggested by a comparison of the somewhat inaccurate early results. The temporal behaviour is shown in Figure 5. Some erratic behaviour is apparent if the accuracy of the

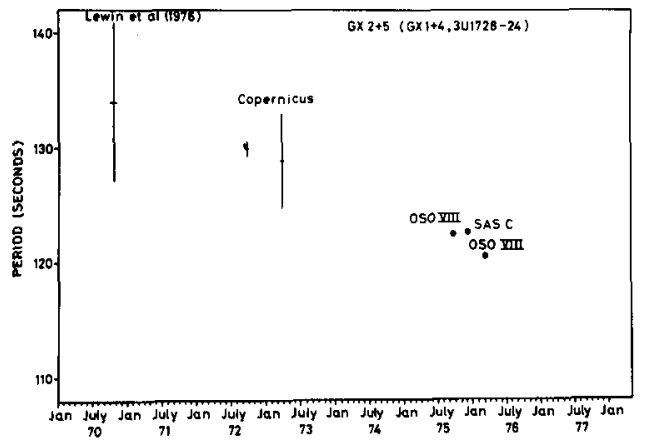

Figure 5. GX1+4 period versus time.

OSO and SAS measurements is as stated, although there is no evidence for any periodic variability in the intrinsic pulse period.

An optical candidate has been suggested for this system by Glass and Feast (1973) and Davidson et al. (1976a, b), but because of the faintness of the object $(V \sim 19)$, only time averaged spectral work has thus far been possible.

Vicinity of $M \times B \quad 1730-33$

This section is deliberately vaguely captioned, since the main results I want to consider come from instruments which have several sources (including at least two burst sources) in the field of view. White et al. (1976a, b) have reported a periodic modulation of the $X$-ray signal when the $3^{\circ}$ FWHM Copernicus detector was pointing at 3U1727-33 on 14th April 1973. The modulation had a period of 7 M90 \pm 0Ma3 with peak to mean amplitude 23 percent of the mean flux in the 2.5 to 7.5 $\mathrm{keV}$ energy band. Because of the 1T45 sampling frequency, they could not rule out various periods below 2 mins. The modulation was very clearly observed throughout a $5 \frac{1}{2}$ hour stretch of data, as can be seen from Figure 6 . The actual average count rate observed was $\sim 3$ times the peak flux given in the Uhuru catalogue; whether this is because 3U1727-33 had brightened since Uhuru observed it, or Copernicus was observing a pulsing transient source similar to A1118-61 is not known. The Ariel 5 experiment $C$ observed the same region of sky for 3 days beginning March 8th 1976, and also for 1 day on March 24th 1976; data were obtained with $\frac{1}{2}, 20$ and 32 seconds time resolution covering the energy band 2 - $7 \mathrm{keV}$. A power spectrum analysis of all the data 


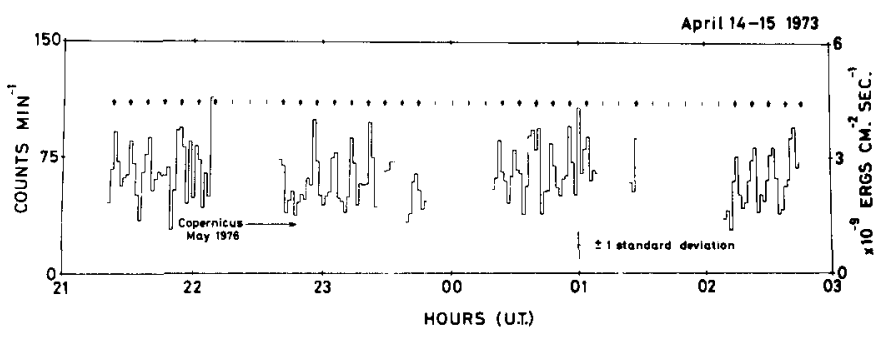

Figure 6. 3U1727-33. Copernicus count rate versus time.

showed no significant periodicities in the range 45 minutes to 40 seconds, with an upper limit of $4 \%$ of the mean flux. However, when the analysis was done on an individual satellite orbit basis, a significant periodicity was detected for one of the orbits with 20 seconds time resolution. The period was $73.1+1.4$ sec with a probability of random occurrence of 10-13. This is a close coincidence with one of the possible aliased periods in the Copernicus data. Copernicus again observed this region on May 16th 1976, when the mean count rate was $250 \%$ of the value observed on April 14th 1973. No periodicity was seen in the range 40 mins to 40 seconds, with an upper limit of $12 \%$ of the mean flux. However, if a transient periodicity such as that seen by Ariel 5 had been present, it would not have been detected because of the low statistical significance of the data. Thus it was concluded that either (a) the 1973 periodicity was due to a regularly pulsating source. Since the periodicity was not present in 1976 either the pulsing was due to a transient source similar to $A 1118-61$, or else the amplitude of modulation is variable. The 73 sec period seen in March 1976 could be the fundamental period, or its apparent relation to the Copernicus period is coincidental. or (b) the 1973 modulation was due to a periodic sequence of bursts from one of the bursters in the field of view, the time between bursts being $7.9 \mathrm{~min}$. or any of its aliases. MX1730-335 has shown quasi-periodic burst trains with inter-burst times at various values between 15 and 35 seconds (Lewin et al. 1976a; Mason et al. 1976a). The 1973 data would require burst trains that are stable for at least 40 pulses if the repeat time is 7.9 mins, or 260 pulses if the bursts repeat every 1.2 min. The regular burst trains seen by Mason et al. lasted for a maximum of about 10 bursts per sequence, and the burst repeat time differed from train to train.

\section{CONCLUDING REMARKS}

Some final comments are now in order. I have deliberately avoided any discussion of the individual $X$-ray source spectra, or mechanisms for their periodicities. Although rotating neutron stars are probably accepted by most people as being the underlying mechanism for the short period sources moving in binary orbits, the situation is not so clear in 
those cases where we have no measureable orbital parameters to give us a guide to the compact object's mass.

As far as source spectra are concerned, it has been pointed out (Maraschi et al. 1976; Jones, 1976) that there exists an observational association between hard (or high temperature) spectra, pulsation, and early type optical counterparts. Maraschi et al. attribute the absence of pulsations in soft $X$-ray sources to the fact that photons produced at the surface of a magnetic neutron star are compton scattered in a surrounding cloud, which is heated by the Compton energy losses of the hardest photons. One glaring anomaly in this respect is 3U1700-37, which, although it is clearly a compact object in a binary orbit, and has a high temperature, has not shown any evidence for pulsations.

There are a variety of ways one could explain this result. Elsner and Lamb (1976) have published a model to account for the complex pulse shapes exhibited by the long period pulsing sources $340900-40$ and A0535+26. It may be that for 3U1700-37 a similar complex pulse shape exists, but that the time structure is much more rapid than the phase resolution of the instrumentation used. Alternatively, irregular variability may be obscuring the pulse profile. Or it may be that there is an unfavourable orientation of the compact object's spin axis relative to the orbit plane. This would then probably indicate an unusual alignment between the spin axis and the orbital plane (taking other observed systems to define the norm). No doubt you could think of countless other possibilities. I intend to halt here.

\section{References}

Auni, Y. and Bahcall, J.N.: 1975, Ap. J. (Letters), 202, L131. Becker, R.H., Boldt, E.A., et al.: 1976, Ap. J. (Letters), 207, L167. Bord, D.J., Mook, D.E., Petro, L., and Hiltner, W.A.:1976, Ap.J.203,689 Bradt, H., Mayer, W., et al.: 1976, Ap. J. (Letters), 204, L67. Chevalier, C. and Ilovaisky, S.: 1974, Astron. and Astrophys., 35, 407. Chodil, G., Mark, H., Rodriques, R., et al.: 1967, Ap. J., 150, 57 . Cooke, B. and Page, C.: 1975, Nature, 256, 712.

Davidsen, A., Malina, R. and Bowyer, s.: 1976, Goddard Symposium on $X$-ray Binaries, NASA SP-389, p691.

Davidsen, A., Malina, R. and Bowyer, S.: 1976, Ap. J., 203, 448. iDavison, P.J. and Fabian, A.C.: 1976, Mon. Not. R. astr. Soc. (in Press). Deeter, J., Crosa, L., Gerend, D. and Boynton, P.: 1976, Ap. J., 206, 861. Elsner, R.F. and Lamb, F.K.: 1976, Nature, 262, 356.

Fabian, A.C. and Davison, P.J.N.: 1974, Talk given at Trieste meeting on $X$-ray Binaries.

Fabian, A.C., Pringle, J. and Rees, M.: 1973, Nature, 244, 212. Giacconi, R., Murray, S., et al.: 1974, Ap. J. Supp., 27, No. 237. Glass, I.S. and Feast, M.W.: 1973, Nature Phys. Sci., 245, 39. Hawkins, F.J., Mason, K.O. and Sanford, P.W.: 1975, Astrophys. Letters, $16,19$.

Hutchings, J.B., Cowley, A.P., et al.: 1974, Ap. J. (Letters), 191, L101. Jones, C.: 1976, Preprint CFA/HEA 76-144. 
Jones, C.A., Chetin, T, and Liller, W.: 1974, Ap. J. (Letters), 190, L1. Jones, C. and Forman, W.: 1976, Preprint CFA/HEA 76-123.

Lewin, W.H.G., Doty, J., et al.: 1976a, Ap. J. (Letters), 207, L95.

Lewin, W.H.G., Hoffman, J. and Doty, J.: 1976b, Paper at High Energy Astrophysics Division meeting of the AAS at Cambridge, Massachusetts. Lewin, W.H.G., Ricker, G.R. and McClintock, J.E.: 1971, Ap. J. (Letters), $169, L 17$.

Liller, W.: 1975a, IAU Circ., No. 2780.

Liller, W.: 1975b, IAU Circ., No. 2888.

Lucke, R., Yentis, D., et al.: 1976, Ap. J. (Letters), 206, L25.

Maraschi, L., Treves, A. and van den Heuvel, E.P.J.: 1976, Ap.J: (in press). Margon, B., Bowyer, S. and Penegor, G.: 1976, Mon. Not. R. astr. Soc., $176,217$.

Mason, K.0., Bell Burnell, S.J. and White, N.E.: 1976a, Nature, 262, 474. Mason, K.0., White, N.E., et al.: 1976, Mon. Not. R. astr. Soc., 176, 193. Mauder, H.: 1974, IAU Circ., No. 2673.

Mauder, H.: 1976, IAU Circ., No. 2946.

MeClintock, J.E., Rappaport, S., et al.: 1976, Ap. J. (Letters), 206, L99. Middleditch, J. and Nelson, J.: 1976, Ap. J., 208, 567.

Patro, L., Feldman, F. and Hiltner, W.A.: 1973, Ap. J. (Letters), 184, L123.

Primini, F., Rappaport, S., et al.:1976, Ap. J. (Letters) In press. Rappaport, S., Joss, P.C., et al.: 1976a, Ap. J. (Letters), 208, L119. Rappaport, S., Joss, P.C., and McClintock, J.E.: 1976b, Ap. J. (Letters), 206, L103.

Robinson, E.L. and Africano, J.: 1975, IAU Circ., No. 2869.

Rosenberg, F.D., Eyles, C.J., et al.: 1975, Nature, 256, 628.

Schmidt, G.D. and Romanishin, W.: 1975, IAU Circ., No. 2873.

Sterne, T.E.: 1939, Mon. Not. R. astr. Soc., 99, 451.

Stothers, R.: 1974, Ap. J., 194; 651.

Swank, J.H., Becker, R.H., et al.: 1976, Ap. J. (Letters), In press.

Tuohy, I., and Rapley, C.G.: 1975, Ap. J. (Letters), In press.

Ulmer, M.P., Baity, W.A. et al.: 1972, Ap. J. (Letters), 178, L121.

van Paradijs, J.A., Hammerschlag-Hensberge, G., van den Heuvel, E.P.J., et al.. 1976, Goddard Symposium on X-ray Binaries, NASA SP-389,p643. Vidal, N.V.: 1973, Ap. J. (Letters), 187, L81.

Webster, B.L., Martin, W.L., et al.: $\overline{1972}$, Nature, Phys. Sci., 240, 183. White, N.E., Huckle, H.E. et al.: 1976a, IAU Circ., No. 2869.

White, N.E., Huckle, H.E., Mason, K.0., et al.: 1976b, paper B2O of High Energy Astrophysics Division of the AAS at Cambridge, Massachusetts. White, N.E., Mason, K.0., Sanford, P.W., and Murdin, P.: 1976c, Mon. Not. R. astr. Soc., 176, 201.

Wilson, R.E.: 1972, Ap. J. (Letters), 174, L27.

Wilson, R.E. and Wilson, A.T.: 1976, AP. J., 204, 551. 